\title{
Optical and near infrared observations of SN 1998bu*
}

\author{
J. Spyromilio ${ }^{1}$, R. Gilmozzi ${ }^{1}$, J. Sollerman ${ }^{2}$, B. Leibundgut ${ }^{1}$, C. Fransson ${ }^{2}$, and J.-G. Cuby ${ }^{3}$ \\ ${ }^{1}$ European Southern Observatory, Karl-Schwarzschild-Strasse 2, 85748 Garching, Germany \\ e-mail: [jspyromi;rgilmozz;bleibundgut]@eso.org \\ 2 Stockholm Observatory, Albanova, 10691 Stockholm, Sweden \\ e-mail: [jesper;claes] @astro.su.se \\ ${ }^{3}$ Laboratoire d'Astrophysique de Marseille, BP 8, 13379 Marseille Cedex 12, France \\ e-mail: jean-gabriel.cuby@oamp.fr
}

Received 31 March 2004 / Accepted 5 July 2004

\begin{abstract}
Infrared and optical spectra of SN 1998bu at an age of one year after explosion are presented. The data show evidence for the radioactive decay of ${ }^{56} \mathrm{Co}$ to ${ }^{56} \mathrm{Fe}$, long assumed to be the powering source for the supernova light curve past maximum light. The spectra provide direct evidence for at least 0.4 solar masses of iron being present in the ejecta of the supernova. The fits to the data also show that the widths of the emission lines increase with time. Photometric measurements in the $H$-band show that the supernova is not fading during the observation period. This is consistent with theoretical expectations.
\end{abstract}

Key words. stars: supernovae: general - stars: supernovae: individual: SN 1998bu

\section{Introduction}

Type Ia supernovae are believed to be the most prodigious producers of iron group elements and given the explosive nature of the events also the best recyclers of such material. The burning of a large fraction of a white dwarf near the Chandrasekhar limit into ${ }^{56} \mathrm{Ni}$ provides the energy for the explosion and subsequent radioactive decay of the nickel to cobalt and then to stable iron provides the energy for the light curve. Type Ia supernovae are popular distance indicators as they exhibit, following a simple calibration procedure, remarkable uniformity (see Phillips et al. 1993). However, our understanding of these objects remains limited (Leibundgut 2001).

The observation of the radioactive decay and accurate direct measurements of the mass of ${ }^{56} \mathrm{Ni}$ manufactured in the explosion provides for a better underpinning of the theoretical scenario outlined above. Evidence for radioactive decay comes from a number of sources. The light curve shape foremost suggests an exponentially declining power source. Kuchner et al. (1994) showed that the flux ratio of a group of [Fe III] lines to [Co III] increased with time in accordance with the expectations of radioactive decay. Schönfelder et al. (1996) report on what would be the most direct and convincing evidence for radioactive decay, namely the direct detection of the $\gamma$-ray lines originating from the decay. However, even for the very luminous SN 1991T the detections are only marginal, although consistent with the current theories. For SN 1998bu

* Based on observations collected at the La Silla and Paranal observatories of the European Southern observatory, Chile in time allocated to proposals $63 . \mathrm{H}-0527,63 . \mathrm{H}-0649$.
Georgii et al. (2002) report upper limits for the amount of ${ }^{56} \mathrm{Ni}$ manufactured in the explosion from the absence of ${ }^{56} \mathrm{Co} \gamma$-ray emission. Their $2-\sigma$ upper limit is $0.35 M_{\odot}$ of ${ }^{56} \mathrm{Ni}$.

The determination of the mass of ${ }^{56} \mathrm{Ni}$ manufactured in the explosion based on optical and infrared spectroscopy has been the subject of many papers and almost as many models. In this paper we present data collected in three observing runs one year after the explosion of the supernova 1998bu. Two epochs of infrared (IR) data are used to explore the evolution of the radioactive element abundances. The later epoch is combined with optical data to derive some limits on the mass of iron present in the ejecta of SN 1998bu.

\section{Observations}

Supernova 1998bu was discovered in NGC 3368 (Villi 1998) on May 9, 1998 and reached maximum light in the $B$-band on May 21st 1998. The supernova has been observed extensively by a number of groups (Suntzeff et al. 1999; Jha et al. 1999; Hernandez et al. 2000). NGC 3368 (M 96) has a distance modulus of $30.25 \pm 0.19$ measured using Cepheid variables (Tanvir et al. 1999) and a recession velocity of $900 \pm 50 \mathrm{~km} \mathrm{~s}^{-1}$. The extinction towards the supernova is high with an $A_{\mathrm{V}}=0.94 \pm 0.15$ (Jha et al. 1999, see also Hernandez et al. 2000 who derive an $A_{\mathrm{V}}$ of $1.0 \pm 0.1$ ).

SN 1998bu was observed with the SofI infrared camera spectrograph at the 3.58-m ESO New Technology telescope at La Silla on January 26, 1999 at an age of 250 days (past maximum blue light). Two grism settings were used providing a spectrum covering the $J, H$ and $K$ bands (Fig. 1). 


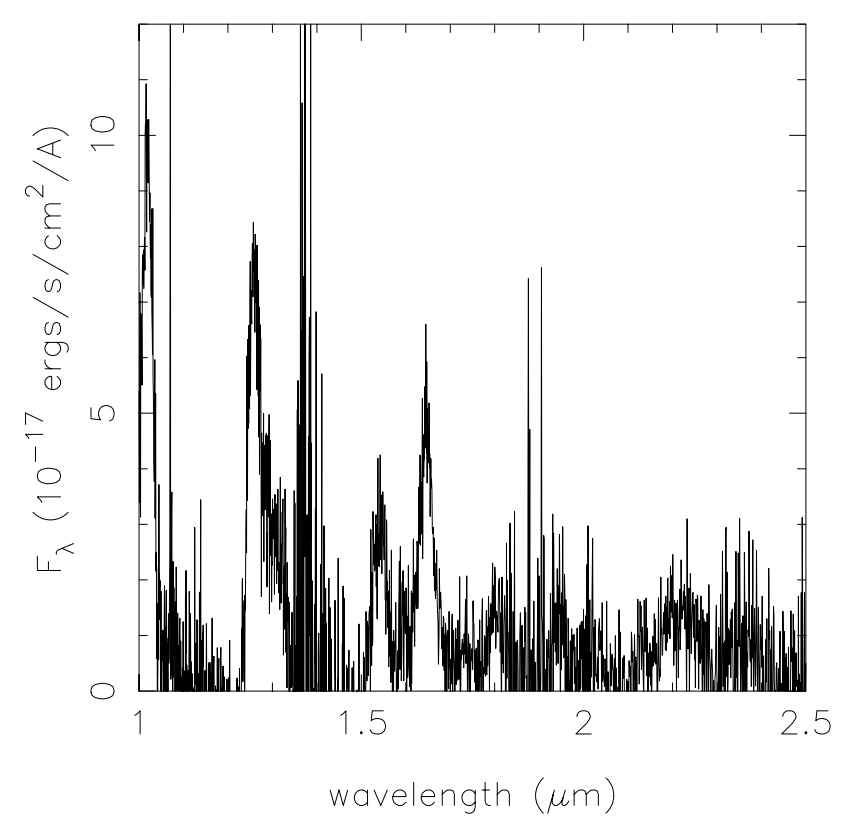

Fig. 1. Spectrum of SN 1998bu obtained with SofI at the NTT on January 26, 1999.

The spectroscopic standard BS4903 was used to remove telluric features. The spectral resolution of the data is $(\lambda / \delta \lambda)=$ 500. The absolute fluxing of the data was based on the photometry obtained on the same night with the same instrument in the $H$ band. The photometric standards were P550C and S217D (Persson et al. 1998). The supernova $H$-band magnitude was $17.5 \pm 0.1$.

The ISAAC infrared camera spectrograph at the 8.2-m ESO UT1 telescope of the VLT array at Paranal was used to observe SN 1998bu on May 1, 1999 at an age of 344 days. Imaging observations were obtained with ISAAC to photometrically calibrate the spectrum. The photometric standard was S860D (Persson et al. 1998) and the derived $H$-band magnitude was $17.5 \pm 0.1$. The spectrum obtained covers the $1.2-1.9-\mu \mathrm{m}$ wavelength range (Fig. 2). The spectral resolution of the data is $(\lambda / \delta \lambda)=1500$. The observations were obtained in two wavelength settings and the spectra were joined without any scaling. The accuracy of merging spectra obtained in different settings without scaling can be confirmed during the fitting of the data. The $1.644-\mu \mathrm{m}$ and $1.257-\mu \mathrm{m}$ lines of [Fe II] originate from the same upper level and their ratio is fixed by the radiative transition rates and the extinction only. As will be shown below the fits are excellent adopting standard atomic data and the commonly assumed extinction for SN 1998bu.

FORS1 observations of SN 1998bu at the 8.2-m ESO UT1 telescope of the VLT array were obtained on June 6, 1999 at an age of 381 days (past maximum blue light). An optical spectrum covering the $0.4-0.9-\mu \mathrm{m}$ wavelength range was obtained. The spectral resolution of the data is $(\lambda / \delta \lambda)=440$. The spectrophotometric standard EG274 was used to flux the spectra. The absolute flux of the spectrum was also checked against the photometry derived from the acquisition images. The night was non-photometric and we have used the local standards as established by Hernandez et al. (2000) (stars 2, 3, 4 and 5) as

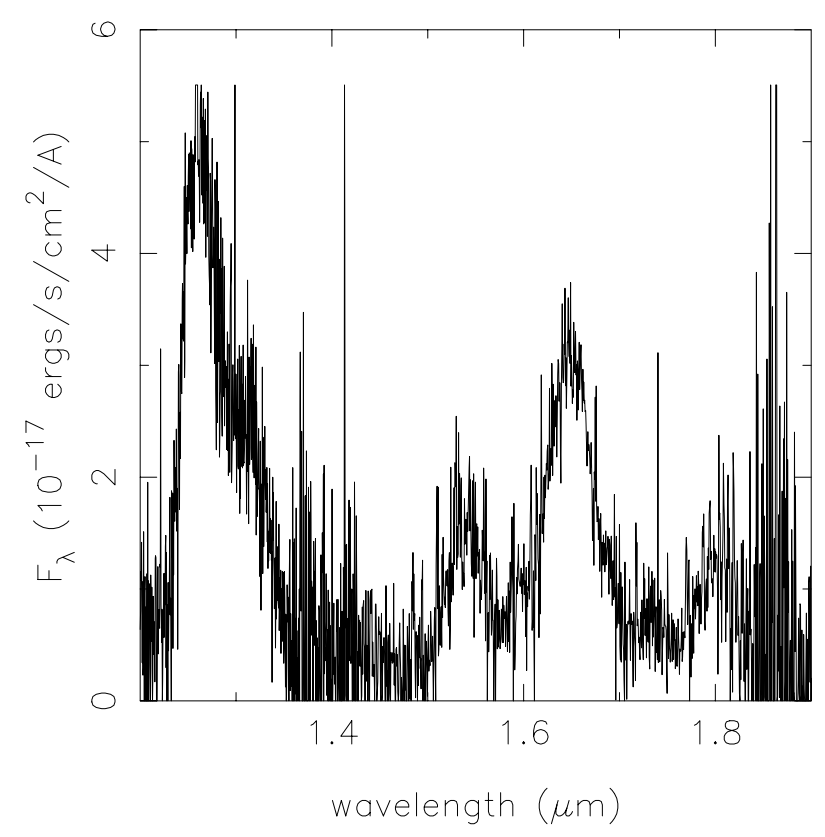

Fig. 2. Spectrum of SN 1998bu obtained with ISAAC at UT1 on May 1, 1999.

well as star 6 of Jha et al. (1999) to derive the SN magnitude. The scatter in the 5 local standards was less than 0.05 mag and no colour terms were applied. The $V$-band magnitude of the supernova was $19.96 \pm 0.10$.

\section{Discussion}

\subsection{The $H$-band light curve}

The observational prescription for IR light curves of type Ia supernovae is that they decline by 0.013 mag per day and comes from the work of Elias \& Frogel (1983) and the more recent update by Meikle (2000). However, these works do not extend their coverage significantly into the first year after explosion. Extrapolating this rule, supernova 1998 bu when observed with ISAAC should have been 1.2 mag fainter than observed with SofI. This is not consistent with our observations (see Fig. 3).

To double-check our zero-points and the accuracy of the photometry we performed photometry of 26 stars common in the field of view for the two instruments. The chosen stars all had magnitudes within \pm 1.5 mag of the supernova, and were not significantly contaminated by the host galaxy. For the stars of comparable brightness to the supernova we derive the same magnitude in both the ISAAC and SofI data with a scatter consistent with the photometric errors in each observation. There is no systematic offset between the two imaging data sets and it is clear that within the errors of the photometry the supernova has not faded between the two observations $( \pm 0.1 \mathrm{mag})$.

The possibility that a fraction of the flux is coming from an echo (reflected light from earlier times that is contributing to the observed magnitude of the supernova) is examined below. However, the late emission line spectrum is not consistent with a significant contribution from an echo. 


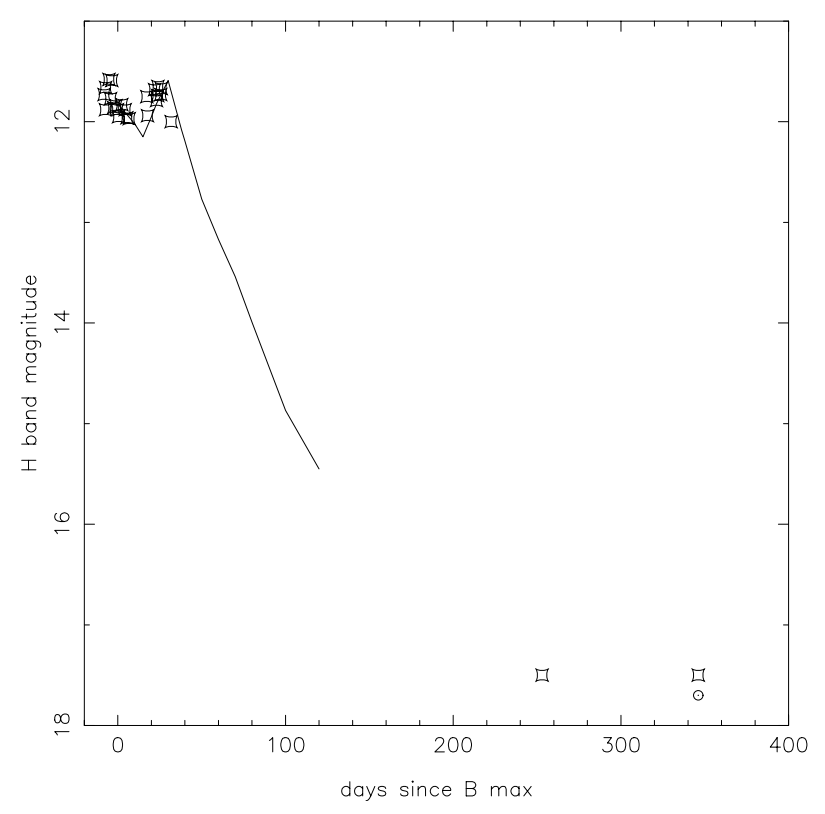

Fig. 3. Light curve of SN 1998bu in the $H$-band. Early data from Meikle (2000) and template light curve from Elias (1983). For the observation at the epoch of 350 days the lower point (dotted circle) indicates the magnitude of the supernova after the removal of a possible contribution from continuum not arising from the ejecta.

\subsection{Removing the echo}

Cappellaro et al. (2001) have demonstrated that the optical spectrum of SN 1998bu at an age of 600 days is dominated by an echo. HST observations have resolved a ring surrounding the supernova which is consistent with emission from an echo. Garnavich et al. (2001) from HST data determine the magnitude of the echo to be 21.4 in the visible (a factor of 2 higher than that shown in the Cappellaro et al. spectrum). We traced the echo spectrum from Cappellaro et al. and have smoothed and scaled it by the HST photometry. It is reproduced together with our FORS1 spectrum in Fig. 4. At the epoch of the FORS1 spectrum no significant continuum emission is expected from the supernova ejecta. The scaled spectrum fits well under the FORS1 data and in all fits to the data we have removed this contribution from our data.

The SofI data, obtained at a time when no contribution from the echo was seen in the Cappellaro light curve, also show no evidence of a continuum. The ISAAC data, however, do show evidence for an underlying continuum at a level of $3 \times 10^{-18} \mathrm{erg} \mathrm{s}^{-1} \mathrm{~cm}^{-2} \AA^{-1}$. Without a detailed model of the dust and its scattering properties it is difficult to establish the exact contribution to this continuum by the echo. At the epochs under consideration in this paper, there is no evidence from other supernovae for a continuum due to emission from the ejecta. A level of $3 \times 10^{-18} \mathrm{erg} \mathrm{s}^{-1} \mathrm{~cm}^{-2} \AA^{-1}$ is somewhat higher than a simple extrapolation of the echo spectrum scaled as mentioned above. However, given the broader $H$-band light curve it is reasonable to expect the IR echo to be somewhat brighter than the optical one. We adopt this continuum level as a non-ejecta contribution.

Adjusting the supernova magnitude for this continuum results in a $\mathrm{H}$-band magnitude of 17.7 for the supernova ejecta at the epoch of the ISAAC observations. We adopt the continuum subtracted spectrum as the one originating from the ejecta. Our conclusions are only marginally affected if we have overestimated the contribution from the echo in the IR spectrum.

\subsection{Fitting the spectra}

As discussed in the introduction a number of authors have used the forbidden lines of iron in supernovae to derive masses in the ejecta. Due to a paucity of data at epochs of one year in the near infrared almost all studies have concentrated on the optical regime (see for example Ruiz-Lapuente \& Lucy 1992; Ruiz-Lapuente et al. 1995; Liu et al. 1997). Spyromilio et al. (1992) did analyse the spectra of SN 1991T covering a broad wavelength range. The optical regime is affected by the reddening to a significant extent and in the absence of IR data other authors have often also fitted the extinction. With the long baseline provided by the IR observations the constraints on the fitting are much stronger. However, the sensitivity of the longer wavelength transitions to temperature and electron density is lower. The combination of optical and IR data constrains the fit significantly.

To combine the ISAAC and FORS1 data into a single spectrum we have first removed the echo spectrum from the FORS1 and ISAAC data following the prescriptions discussed above. We have then increased the flux in the FORS1 data by 0.0138 mag per day (measured on the published $V$-band light curve, excluding the echo contribution) making up for the 41 days of difference in the epoch of the FORS1 and ISAAC data.

Our modeling is based on the techniques and models described in Spyromilio et al. (1992). The line identifications are the same as those of Liu et al. (1997) for the optical lines and those of Spyromilio et al. (1992) for the infrared lines. The temperatures and densities for our models are of order $4000 \mathrm{~K}$ and $2 \times 10^{5} \mathrm{~cm}^{-3}$ for the SofI epoch data and the same temperature but lower electron density $\left(8 \times 10^{4} \mathrm{~cm}^{-3}\right)$ for the ISAAC epoch. For simplicity cobalt and nickel are treated in LTE. Since we only derive abundances from iron lines we treat these in NLTE. The atomic data are from Nussbaumer \& Storey (1988a), Nussbaumer \& Storey (1980), Garstang (1957), Berrington et al. (1991), Grevesse et al. (1971), Nussbaumer \& Storey (1988b).

From the clean $1.644 \mu \mathrm{m}$ line of [Fe II] we can derive a mass of iron emitting given the emissivity from the NLTE calculations used above. Using a distance of $11 \mathrm{Mpc}$ for M 96, $A_{\mathrm{V}}=0.94$, and an observed flux from the $1.644 \mu \mathrm{m}$ line of $1.7 \times 10^{-14} \mathrm{erg} \mathrm{s}^{-1} \mathrm{~cm}^{-2}$ then the mass of singly ionised iron present in the ejecta is derived to be $0.4 M_{\odot}$ with a conservative error of $0.1 M_{\odot}$. The value depends solely on the emissivity for this transition. Doubly ionized iron has strong lines in the optical. However, the [Fe III] lines are heavily blended and are of higher excitation making their emissivity less reliable. A good fit to the [Fe III] lines could be achieved with a variety of conditions. We do not believe that from our models a more accurate mass of $\mathrm{Fe}^{++}$can be derived. We leave that to more sophisticated modeling attempts in a future paper. 


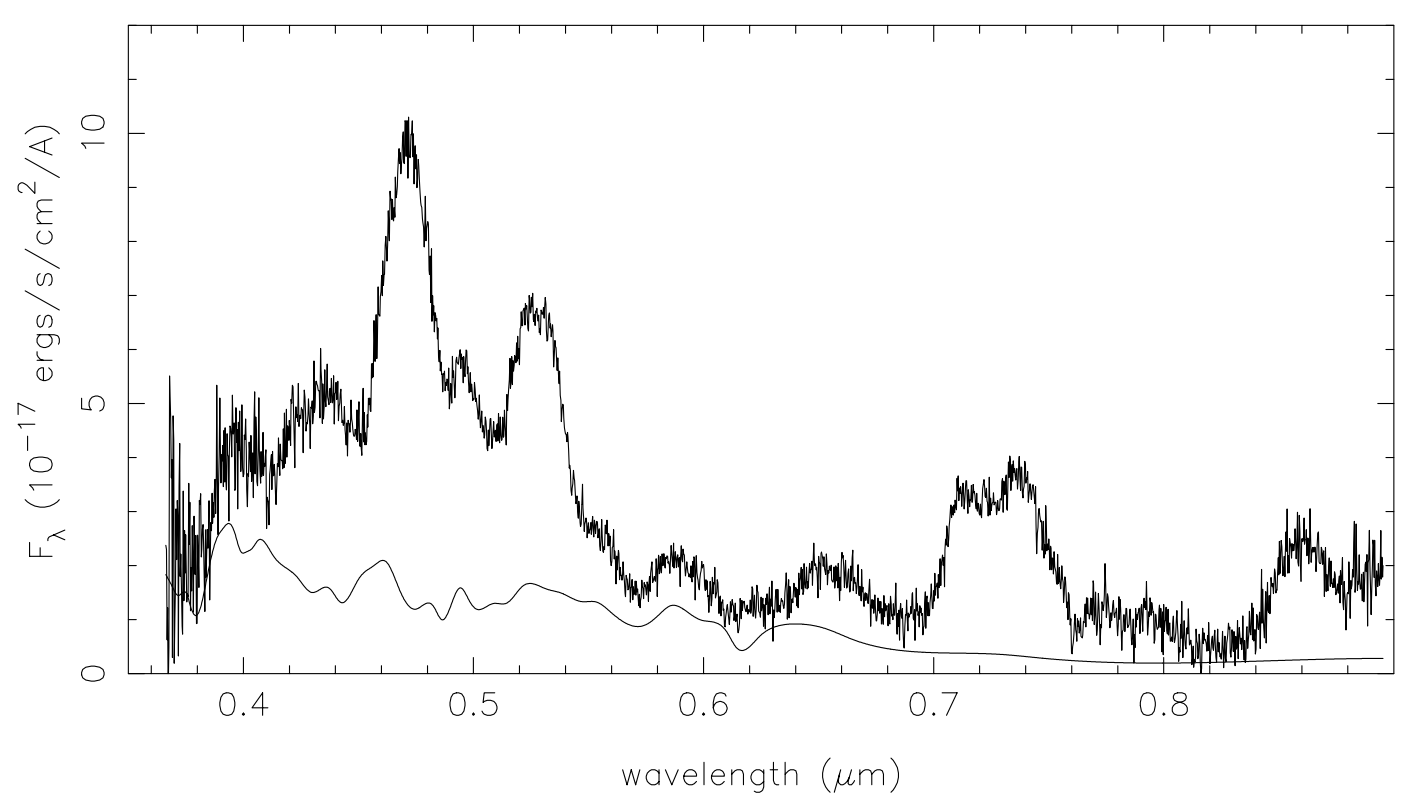

Fig. 4. Spectrum of SN 1998bu obtained with FORS1 at UT1 on June 11, 1999. The lower spectrum is a trace of the late light echo spectrum (Cappellaro et al. 2001) scaled by a factor of 2 upwards - see text for details.

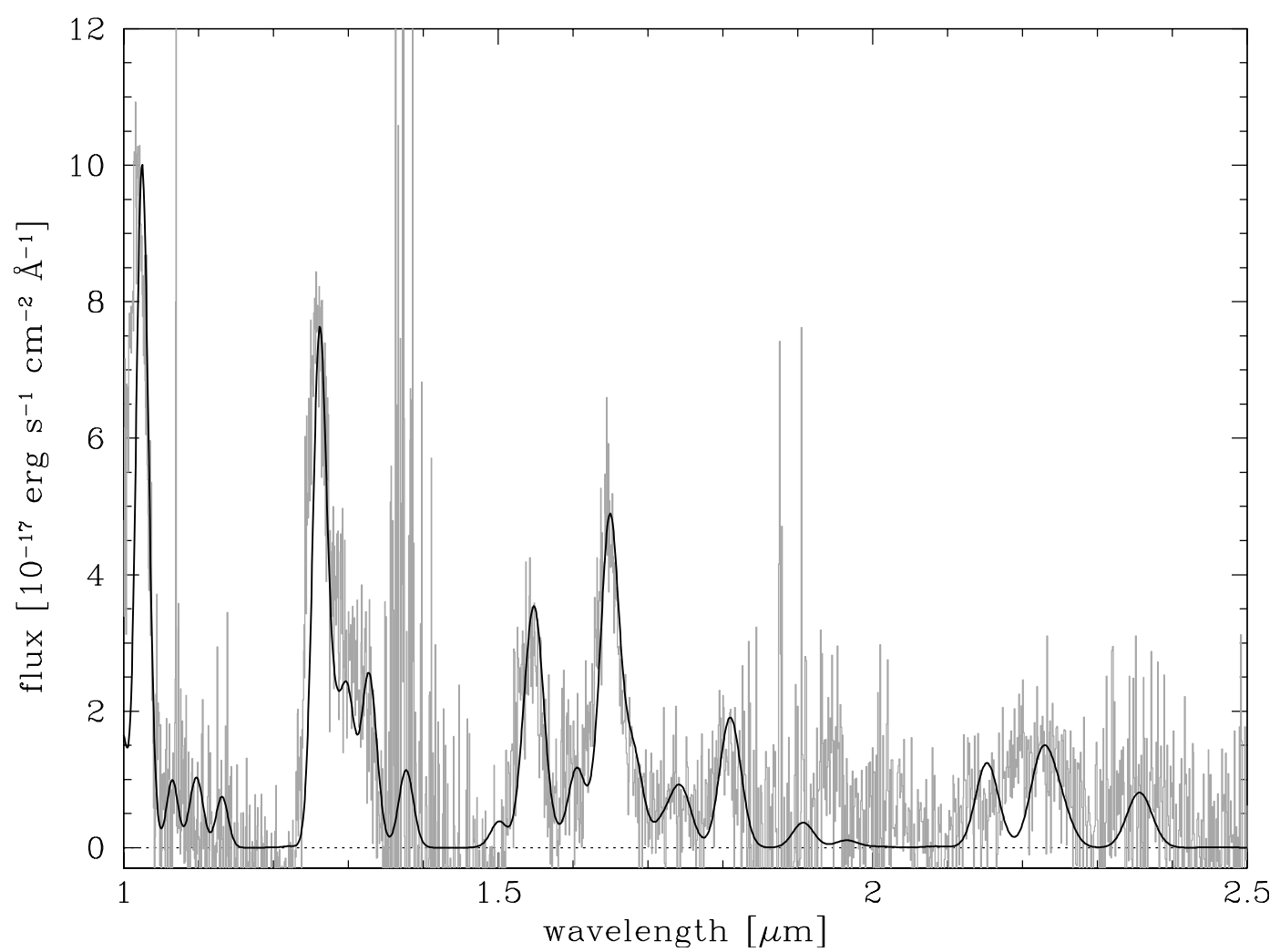

Fig. 5. A fit to the spectrum obtained with SOFI at the NTT using a simple NLTE [Fe I], [Fe II] and [Fe III] model and LTE [Co II]. The narrow (few pixel) spikes in the data are artifacts of the atmosphere and/or the detector.

Contardo et al. (2000) have analyzed the light curves of a number of type Ia supernovae and find typically $0.5 M_{\odot}$ of ${ }^{56} \mathrm{Ni}$ to have been manufactured in the explosion. This is consistent with our result for SN 1998bu, but only marginally consistent with the claimed upper limit from absence of the $\gamma$-ray line emission (Georgii et al. 2002; Milne et al. 2004).
Fransson et al. (1996) predicted from theoretical modeling that the near-infrared lightcurves would flatten out a year after explosion. As discussed earlier we observe this effect. The mass of iron we observe emitting is inconsistent with this being the classical infrared catastrophe as described by Axelrod (1980) whereby the flattening of the cooling curve combined 


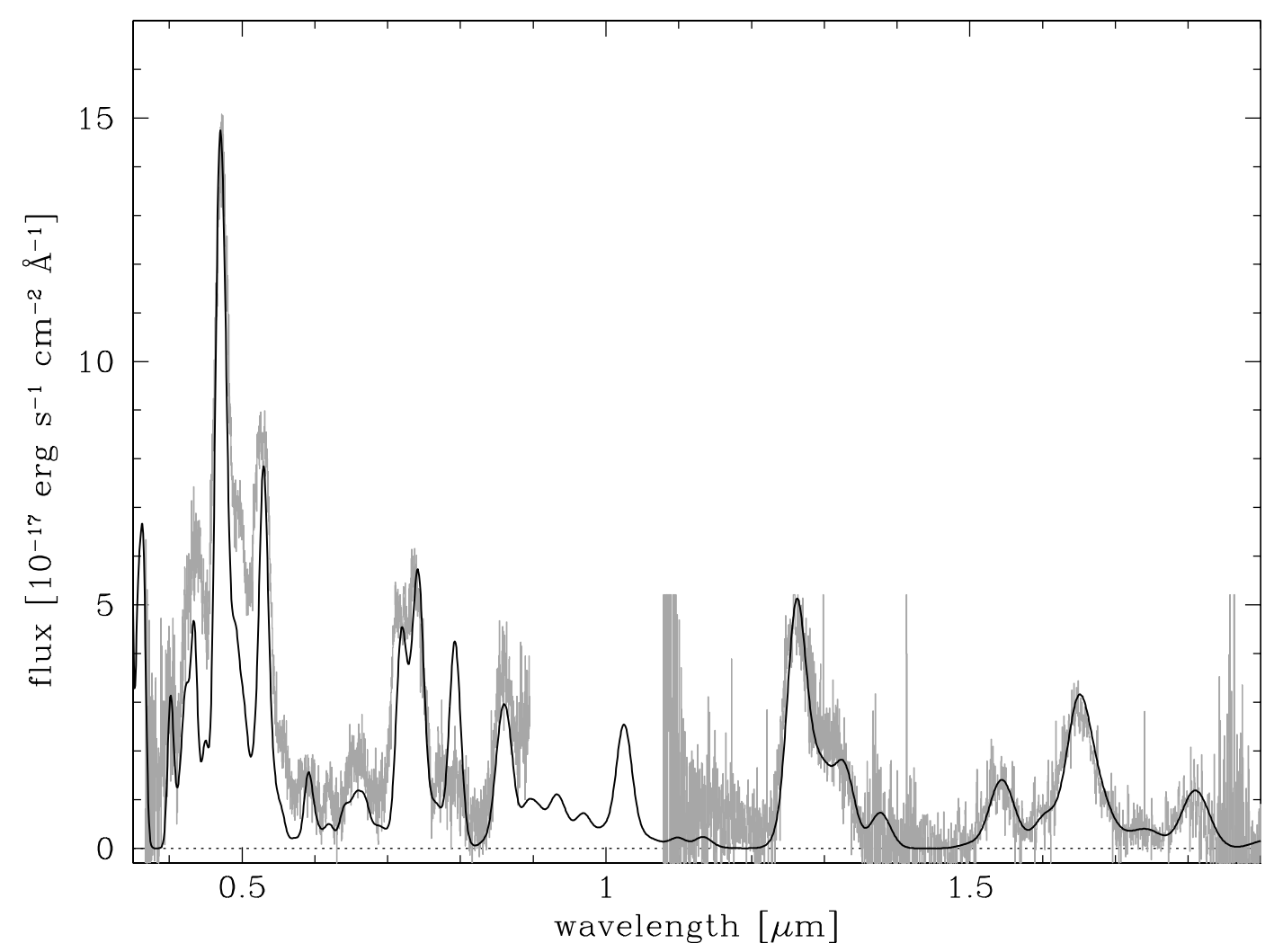

Fig. 6. A fit to the combined FORS1 and ISAAC spectrum using a simple NLTE [Fe I], [Fe II] and [Fe III] model and LTE [Co II], [Co III], [Ni II], [Ni III]. The FORS1 spectrum has been scaled to match the ISAAC one based on the photometric evolution (see text for details). The narrow (few pixel) spikes in the data are artifacts of the atmosphere and/or the detector.

with the exponential decline of the heating due to the radioactivity combine to produce a rapid cooling of the ejecta resulting in only ground state energy levels being populated. Midinfrared transitions would remain observable but higher energy lines should vanish. In SN 1987A the infrared catastrophe was seen without the disappearance of the optical and near-IR lines. However, only a small fraction of the mass of iron was emitting in these lines. In our data presented here this is not the case. A significant amount of iron is still emitting in the optical and near-IR.

The flattening of the light curve may be due to the gradual transition of the emission from the optical to the near-IR as the ejecta expand and the densities drop. The electron densities remain high enough that the ejecta continue to be coupled to the heating source.

We note that also the type Ia SN 2000cx displayed a constant late light curve in the near infrared. The flat lightcurve was observed in the $J$ - and $H$-bands between 360-480 days past maximum, and appears consistent with late time modeling (Sollerman et al. 2004a).

\subsection{Line widths and profiles}

From the fitting of the spectra we detect a change in the line widths of the emission lines. After correction for the recession velocity of the parent galaxy we find that a Gaussian profile fits the observed emission well on the red side of each line. The Gaussian profiles do not produce a good fit to the blue side of the emission lines (see Figs. 7 and 8). The supernova seems to emit more in the blue than in the red.

Since the lines we are observing are forbidden and moreover, are emitted in a rapidly expanding medium, self absorption is not considered. However, a skewed distribution of the emitting material could easily explain the line profiles.

The velocities required for the fits were $5000 \pm 200 \mathrm{~km} \mathrm{~s}^{-1}$ for the SofI data and $7000 \pm 200 \mathrm{~km} \mathrm{~s}^{-1}$ for the ISAAC data. The errors on the velocities are conservative as multiple lines are fit. This increase in the observed emitting volume implies that the outer regions of the ejecta increased their relative emissivity with respect to the centre between the two epochs. The excess emission in the blue part of the profile means that the width of the Gaussian is constrained by the red side of the line and the expansion velocity is a minimum. A broader skewed line profile would provide a better fit. Since all 3 instruments were wavelength calibrated independently we exclude a calibration error as the source of the effect seen.

The change of the expansion velocities observed suggests that the results of Mazzali et al. (1998) relating the observed late-time expansion velocity to the energy of the explosion should be reconsidered as the measured velocity may depend on the exact epoch of observation.

\subsection{Direct evidence for radioactive decay}

Varani et al. (1990) demonstrated in the case of SN 1987A that the near IR lines of [Co II] and [Fe II] at 1.547 and $1.533 \mu \mathrm{m}$, 


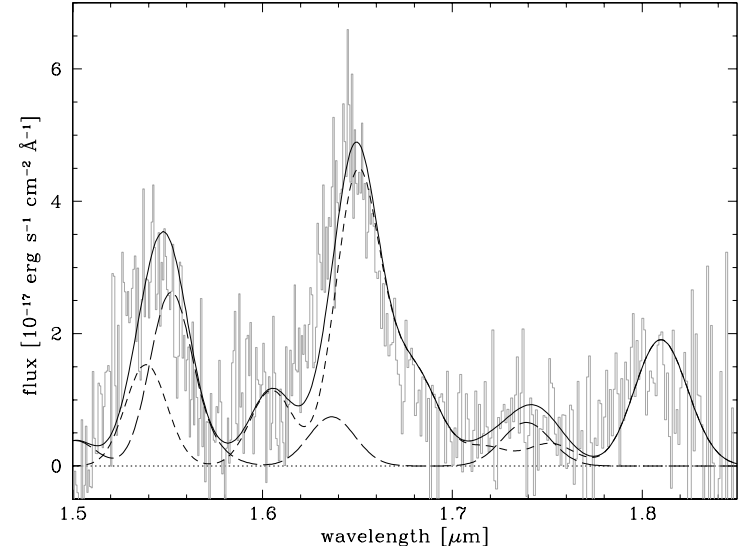

Fig. 7. Same as Fig. 5 but focused on the $H$-band. The long dashed line marks the [Fe II] spectrum and the short dashed line the [Co II]. The continuous line is the sum. The $1.55-\mu \mathrm{m}$ feature is fit by the $1.533[\mathrm{Fe} \mathrm{II}]$ and 1.547 [Co II] lines.

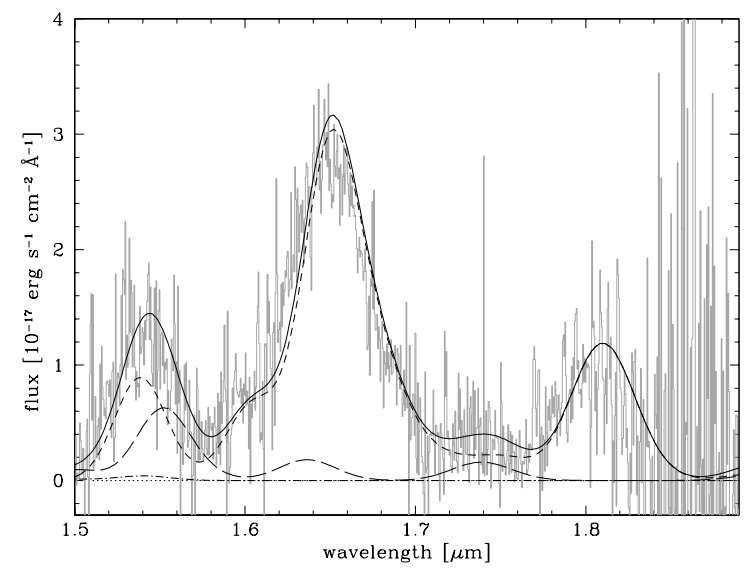

Fig. 8. Same as Fig. 6 but focused on the $H$-band. The spectra identification is as per Fig. 5. Additionally the dot-dash line marks the [Fe I] spectrum. The $1.55-\mu \mathrm{m}$ feature is fit by the 1.533 [Fe II] and 1.547 [Co II] lines.

respectively, could be used to determine the ratio of the mass of iron to cobalt emitting in the ejecta of a supernova. The ratio of the two lines changes as radioactive cobalt decays into stable iron. The two lines arise from the same ionization stage and their excitation potentials are very similar. The ratio of the two lines is very weakly dependent on temperature and it is expected that the ionization structure of the cobalt and iron will be very similar. Therefore the ratio of the lines is expected to directly relate to the ratio of the masses of iron to cobalt.

The following formula was derived by Varani et al. (1990) for the mass fraction:

$\frac{M_{\mathrm{Co}}}{M_{\mathrm{Fe}}}=\frac{F_{1.547}}{F_{1.533}} \times 0.0376 \times \mathrm{e}^{\frac{2127}{T}}$

where $T$ is the temperature of the gas in degrees Kelvin (4000 K in our case). From the fits to the data (see Figs. 7 and 8) we derive the ratios and plot them as a function of age in Fig. 9.

Superimposed on the two data points is the change of the mass ratio of cobalt to iron as expected from the radioactive decay. A pure ${ }^{56} \mathrm{Ni}$ initial mass is assumed for this plot with no contribution by ${ }^{57} \mathrm{Ni}$. The optical cobalt and iron lines have

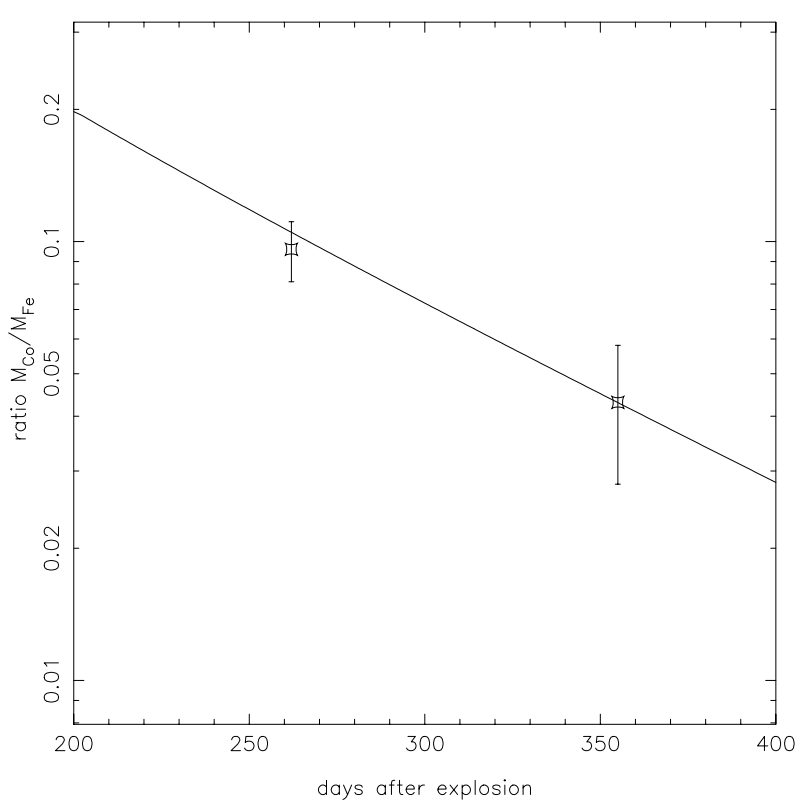

Fig. 9. The derived mass ratio of Co to Fe in the ejecta of SN 1998bu as a function of time after explosion. The line represents the theoretical evolution from radioactive decay and is not fit to the data. The error bars reflect a error of $1000 \mathrm{~K}$ in the assumed temperature for the electron gas.

been used in the past by other authors (see Kuchner et al. 1994) to derive similar results for type Ia supernovae.

\section{Conclusions}

We have used late time optical and infrared photometry and spectroscopy of the type Ia supernova 1998bu to determine the physical conditions in the ejecta a year after the explosion. The mass of emitting iron is consistent with determinations by other methods and in agreement with theoretical predictions. We find that the supernova is fading very slowly in the $H$-band one year after the explosion.

We find that the emission lines originating within the ejecta appear broader and skewed at later times possibly indicating an assymetric distribution of iron within the ejecta. The widths of the emission lines also increases with time. The emission observed in the infrared [Fe II] lines is consistent with 0.4 solar masses of $\mathrm{Fe}^{+}$being present in the ejecta. The evolution of the spectrum in the near infrared shows direct evidence of the radioactive decay of ${ }^{56} \mathrm{Co}$ into ${ }^{56} \mathrm{Fe}$.

Acknowledgements. We thank the staff at the NTT for their excellent support over many years of observing. The ISAAC observations were obtained in service mode and we thank the Paranal staff for doing our observing for us. This research has made use of the NASA/IPAC Extragalactic Database (NED) which is operated by the Jet Propulsion Laboratory, California Institute of Technology, under contract with the National Aeronautics and Space Administration.

\section{References}

Axelrod, T. S. 1980, Ph.D. Thesis, Univ. California, Santa Cruz Berrington, K. A., Zeippen, C. J., Le Dourneuf, M., et al. 1991, J. Phys. B, 24, 3467 
Cappellaro, E., Patat, F., Mazzali, P. A., et al. 2001, ApJ, 549, L215

Contardo, G., Leibundgut, B., \& Vacca, W. D. 2000, A\&A, 876

Elias, J. H., \& Frogel, J. A. 1983, ApJ, 268, 718

Fransson, C., Houck, J., \& Kozma, C. 1996, in Supernovae and supernova remnants, ed. R. McCray, \& Z. Wang (Cambridge University press), IAU Coll., 145, 211

Garnavich, P. M., Kirshner, R. P., Chalis, P., et al. 2001, BAAS, 33, 1370

Garstang, R. H. 1957, MNRAS, 117, 393

Georgii, R., Plüschke, S., \& Diehl, R., et al. 2002, A\&A, 394, 517

Grevesse, N., Nussbaumer, H., \& Swings, J. P. 1971, MNRAS, 151, 239

Hernandez, M., Meikle, W. P. S., Aparicio, A., et al. 2000, MNRAS, 319,223

Jha, S., Garnavich, P. M., Kirshner, R. P., et al. 1999, ApJS, 125, 73

Kuchner, M. J., Kirshner, R. P., Pinto, P. A., \& Leibundgut, B. 1994, ApJ, 426, L89

Leibundgut, B. 2001, ARA\&A, 39, 67

Liu, W., Jeffery, D. J., \& Schultz, D. R. 1997, ApJ, L107

Mazzali, P. A., Cappellaro, E., Danziger, I. J., Turatto, M., \& Benetti, S. 1998, ApJ, 499, L49

Meikle, W. P. S. 2000, MNRAS, 314, 782

Milne, P. A., Hungerford, A. L., Fryer, C. L., et al. 2004 [arXiv: astro-ph/0406173]
Nussbaumer, H., \& Storey, P. J. 1980, A\&A, 89, 303

Nussbaumer, H., \& Storey, P. J. 1988a, A\&A, 193, 327

Nussbaumer, H., \& Storey, P. J. 1988b, A\&A, 200, L25

Persson, S. E., Murphy, D. C., Krzeminski, W., Roth, M., \& Rieke, M. J. 1998, AJ, 116, 2475

Phillips, M. M. 1993, ApJ, 413, L105

Ruiz-LaPuente, P., \& Lucy, L. B. 1992, ApJ, 400, 127

Ruiz-LaPuente, P., Kirshner, R. P., Phillips, M. M., et al. 1995, ApJ, 439,60

Schönfelder, V., Bennett, K., Bloemen, H., et al. 1996, A\&AS, 120, 13

Sollerman, J., Kozma, C., \& Lindahl, J. 2004a, to appear Supernovae: 10 Years of 1993J, ed. J. M. Marcaide, \& K. W. Weiler, in Proc. of IAU Coll., 192 [arXiv: astro-ph 0311075]

Sollerman, J., Lindahl, J., Kozma, C., et al. 2004b, A\&A, submitted

Spyromilio, J., Meikle, W. P. S., Allen, D. A., \& Graham, J. R. 1992, MNRAS, 258, 53P

Spyromilio, J., \& Graham, J. R. 1992, MNRAS, 255, 671

Suntzeff, N. B., Phillips, M. M., Covarrubias, R., et al. 1999, AJ, 117, 1175

Tanvir, N. R., Ferguson, H. C., \& Shanks, T. 1999, MNRAS, 310, 27

Varani, G.-F., Meikle, W. P. S., Spyromilio, J., \& Allen, D. A. 1990, MNRAS, 245, 570

Villi, M. 1998, IAU Circ., 6899 